\title{
Shikonin inhibits CEBPD downregulation in IL-17-treated HaCaT cells and in an imiquimod-induced psoriasis model
}

\author{
XIAO-OU LAN ${ }^{1,2^{*}}$, HE-XIAO WANG ${ }^{1}$, RUI-QUN QI ${ }^{1}$, YUAN-YUAN XU ${ }^{1}$, YA-JIE YU ${ }^{1,3}$ \\ YANG YANG $^{1}$, HAO GUO $^{1}$, XING-HUA GAO ${ }^{1}$ and LONG GENG ${ }^{1 *}$ \\ ${ }^{1}$ Department of Dermatology, The First Hospital of China Medical University, Shenyang, Liaoning 110001; \\ ${ }^{2}$ Department of Dermatology, The Seventh People's Hospital of Shenyang, Shenyang, Liaoning 110003; \\ ${ }^{3}$ Department of Dermatology, The Fifth Affiliated Hospital of Sun Yat-Sen University, Zhuhai, Guangdong 519000, P.R. China
}

Received September 15, 2019; Accepted June 8, 2020

DOI: $10.3892 / \mathrm{mmr} .2020 .11315$

\begin{abstract}
Psoriasis is a chronic inflammatory skin disease characterized by well-defined scaly papules and plaques. Interleukin (IL)-17 is involved in its pathogenesis and promotes the proliferation of epidermal keratinocytes through signal transducer and activator of transcription 3 (STAT3) activation. Shikonin, a natural naphthoquinone isolated from Lithospermum erythrorhizon, possesses anti-inflammatory and immunosuppressive properties and can suppress IL-17-induced vascular endothelial growth factor expression by inhibiting the JAK/STAT3 pathway. In the present study, MTS, iCELLigence and RT-qPCR were used to determine the optimal concentration and duration of IL-17 or shikonin acting on $\mathrm{HaCaT}$ cells. The changes in the expression levels of genes associated with the IL-6/STAT3 pathway in differentially treated cells were analyzed via $\mathrm{RT}^{2}$ Profiler $^{\mathrm{TM}}$ PCR Array. Small interfering RNA was used to silence the expression levels of the target gene CCAAT/enhancer-binding protein $\delta$ (CEBPD). Western blotting and immunohistochemistry were used to evaluate the effect of shikonin on imiquimod-induced psoriasis in mice and the expression levels of CEBPD. Shikonin reversed IL-17-mediated downregulation of the tumor suppressor CEBPD in HaCaT cells. Moreover, low levels of CEBPD in the imiquimod-induced mouse model of psoriasis were restored by shikonin treatment, which ameliorated excessive keratinocyte proliferation. Taken together, these findings suggest that CEBPD plays a key role in the pathogenesis of psoriasis and can be targeted by shikonin as a potential therapeutic strategy.
\end{abstract}

Correspondence to: Professor Long Geng, Department of Dermatology, The First Hospital of China Medical University, 155 Nanjing Street, Heping, Shenyang, Liaoning 110001, P.R. China E-mail: genglong_sy@163.com

*Contributed equally

Key words: CCAAT/enhancer-binding protein $\delta$, interleukin-17, psoriasis, shikonin, signal transducer and activator of transcription 3

\section{Introduction}

Psoriasis is a chronic T cell-mediated inflammatory skin disease that is driven by overproduction of pro-inflammatory cytokines and excessive proliferation of keratinocytes (1). Interleukin (IL)-17 acts as the primary pathological effector of psoriasis (1-3) by regulating keratinocyte proliferation through signal transducer and activator of transcription 3 (STAT3) (4-6). STAT3 directly binds to IL-17 $(7,8)$ and is also a key regulator of keratinocyte function (5). Consistent with this, STA-21, a small molecule inhibitor of STAT3, significantly alleviates the severity of lesions in patients with psoriasis $(6,9,10)$. Keratin 17 (K17) is a marker of psoriasis that is undetectable in normal epidermis but markedly upregulated in psoriatic lesions $(11,12)$. A recent study has suggested that IL-17 induces K17 in the epidermis by promoting the nuclear translocation of STAT3, which contributes to the pathogenesis of psoriasis (13).

Topical application of corticosteroids is currently the standard treatment option for psoriasis (14). Nevertheless, natural, plant-derived extracts are a promising therapeutic option owing to their fewer side effects. Shikonin is a $288-\mathrm{kDa}$ liposoluble naphthoquinone derived from Lithospermum erythrorhizon, which is used in Traditional Chinese Medicine for the treatment of various diseases, such as breast and prostate cancer, as well as lung adenocarcinoma and hepatocellular carcinoma $(15,16)$. Shikonin displays anti-inflammatory, immunosuppressive and anti-tumor properties (17-20) and inhibits the proliferation of skin cancer cells by blocking STAT3 $(21,22)$. Our previous study demonstrated that shikonin could suppress IL-17-induced production of cytokines associated with psoriasis by inhibiting the JAK/STAT3 signaling pathway (23). Moreover, Liu et al (24) found that shikonin could downregulate K17 in proliferating keratinocytes by interfering with STAT3 signaling. More recently, Yu et al (25) also demonstrated that shikonin induced $\mathrm{G}_{0} / \mathrm{G}_{1}$ cell cycle arrest in the human $\mathrm{HaCaT}$ keratinocyte cell line. However, the potential effects of shikonin on the downstream mediators of the JAK/STAT3 signaling pathway are unclear.

The CCAAT/enhancer-binding protein $\delta$ (CEBPD) has been implicated in physiological processes, such as cell 
differentiation, metabolism, inflammation, cell cycle arrest and apoptosis (26). It is regulated by inflammatory cytokines, such as IL-6, and its protein overexpression can induce cell cycle arrest and apoptosis in several types of cancer, such as prostate cancer, neuroblastoma and acute myeloid leukemia (27-31). Li et al (32) demonstrated that Q-associated kinase 2 knockdown could upregulate CEBPD mRNA and protein expression levels and activity, resulting in increased proliferation of hepatocellular carcinoma cells in vitro and in vivo. In addition, CEBPD is also activated by IL- 6 and IL-17 in response to chemotherapeutic anti-cancer drugs $(33,34)$. Furthermore, CEBPD is also critical for IL-17 and tumor necrosis factor- $\alpha$ (TNF- $\alpha$ )-induced expression of lipocalin 2 , serum amyloid A3 pseudogene and IL-6 during psoriasis progression (35). However, to the best of our knowledge, the mechanisms underlying the effect of CEBPD in keratinocytes remains largely unknown.

The aim of the present study was to analyze the effect of shikonin on the IL-6/STAT3 signaling pathway in IL-17-treated HaCaT cells using the $\mathrm{RT}^{2}$ Profiler $^{\mathrm{TM}}$ PCR Array system. In addition, the effect of shikonin on the imiquimod (IMQ)-induced murine psoriasis model was also evaluated.

\section{Materials and methods}

Cell culture. HaCaT cells (GCC-AO0003CS; Shanghai Jikai Gene Medical Technology Co., Ltd.) were cultured in DMEM (HyClone; GE Healthcare Life Sciences) supplemented with $10 \%$ heat-inactivated FBS (Gibco; Thermo Fisher Scientific, Inc.), $100 \mathrm{U} / \mathrm{ml}$ penicillin (HyClone; GE Healthcare Life Sciences), and $100 \mu \mathrm{g} / \mathrm{ml}$ streptomycin (HyClone; GE Healthcare Life Sciences). The HaCaT cell line was authenticated by the supplier using STR profiling. The cells were maintained in a humidified incubator at $37^{\circ} \mathrm{C}$ with $5 \% \mathrm{CO}_{2}$.

MTS assay. The proliferation of HaCaT cells was assessed using a MTS assay (Promega Corporation) according to the manufacturer's instructions. Briefly, the cells were seeded into 96-well plates, at a density of 5,000 cells/well and cultured for 24,48 and $72 \mathrm{~h}$ in the presence of varying concentrations of shikonin $(0.00,0.01,0.05,0.25,0.50,1.00,2.50$ or $5.00 \mu \mathrm{M})$ (Sigma-Aldrich; Merck KGaA) in a humidified incubator at $37^{\circ} \mathrm{C}$ with $5 \% \mathrm{CO}_{2}$. In order to verify the effect of shikonin on IL-17 induced HaCaT cell proliferation, the HaCaT cells were seeded into 96-well plates, at a density of 5,000 cells/well and cultured in a humidified incubator at $37^{\circ} \mathrm{C}$ for 24,48 and 72 h with IL-17 (PeproTech, Inc.), shikonin, IL-17 + shikonin, or no treatment. In order to verify the effect of shikonin on IL-17-induced HaCaT cell proliferation when CEBPD is silenced, the assay was performed with scrambled siRNA (NC), RNAi + IL-17, RNAi + shikonin, RNAi + IL-17 + shikonin, and untreated cells. The number of cells and the duration of treatment are consistent with the previous description. The absorbance was measured in each well at $490 \mathrm{~nm}$ using a microplate reader (Bio-Rad Laboratories, Inc.). Each sample was analyzed in six replicates, and the assay was repeated three times.

iCELLigence system. The proliferation, viability, and morphology of the cells can be ascertained on the basis of electrical impedance when cultured on micro-electrodes (36). The HaCaT cells were seeded in two iCELLigence system 8-well plates (ACEA Bioscience, Inc.) at a density of 5,000 cells/well in $300 \mu \mathrm{l}$ medium and cultured for $14 \mathrm{~h}$. Then, different concentrations of IL-17 (0, 10, 20, 30, 40, 50 or $60 \mathrm{ng} / \mathrm{ml}$ ) were added and cultured for $56 \mathrm{~h}$ in a humidified incubator at $37^{\circ} \mathrm{C}$. Each sample was tested in duplicate. In addition, the cells were cultured in quadruplicate with $40 \mathrm{ng} / \mathrm{ml}$ IL-17, $1 \mu \mathrm{M}$ shikonin or both for $96.8 \mathrm{~h}$. The cell index (CI), a measure of the number of cells, was monitored in real-time to assess proliferation using RTCA software (37). Each assay was repeated three times.

Reverse transcription quantitative-PCR (RT-qPCR). The $\mathrm{HaCaT}$ cells were cultured with $40 \mathrm{ng} / \mathrm{ml} \mathrm{IL}-17$ for $0,2,4,6,8$ and $10 \mathrm{~h}$ and harvested. Total RNA was isolated using the miRNeasy Mini kit and cDNA was synthesized from RNA using a GoScript ${ }^{\mathrm{TM}}$ Reverse Transcription System (Promega Corporation) according to the manufacturer's instructions. RNA concentrations were obtained using the Nanodrop ND-1000 spectrophotometer (NanoDrop Technologies; Thermo Fisher Scientific, Inc.). The sequences of the primers used were as follows: STAT3 forward, 5'-CACCAAGCGAGG ACTGAGCAT-3' and reverse, 5'-GCCAGACCCAGAAGG AGAAGC-3'; CEBPD forward, 5'-ACTTACCACCACTAA ACTGCGAG-3' and reverse, 5'-CTGCATCAACAGGAG TAAGATGTAG-3'; K17 forward, 5'-CCACCCAGAAGACT GTGGAT-3' and reverse, 5'-TTCTAGACGGCAGGTCA GGT-3'; and GAPDH forward, 5'-TGGAGTCTACTGGCGT CTT-3'; and reverse, 5'-TGTCATATTTCTCGTGGTTCA-3' (all Invitrogen; Thermo Fisher Scientific, Inc.). RT-qPCR was performed in 96-well plates using a 7900HT Fast Real-Time PCR system (Thermo Fisher Scientific, Inc.). The RT ${ }^{2}$ SYBR Green qPCR Master mix (Promega Corporation) was employed for amplification on a reaction mixture containing primers (0.4 $\mu \mathrm{l}$ each), $2 \mathrm{x}$ qPCR Master Mix $(10 \mu \mathrm{l})$, cDNA $(2 \mu \mathrm{l})$, and nuclease free water. Amplification was performed as follows: $95^{\circ} \mathrm{C}(2 \mathrm{~min}) ; 40$ cycles at $95^{\circ} \mathrm{C}(15 \mathrm{sec})$ and $60^{\circ} \mathrm{C}(1 \mathrm{~min})$. The relative mRNA expression levels were calculated via the $2^{-\Delta \Delta \mathrm{Cq}}$ method (38). Each sample was analyzed in six replicates, and the assay was repeated three times.

$R^{2}$ Profiler $^{T M}$ PCR array analysis. Cells were seeded in 6 -well plates at a density of $5 \times 10^{5}$ cells/well and treated with $40 \mathrm{ng} / \mathrm{ml} \mathrm{IL-17,} 1 \mu \mathrm{M}$ shikonin or both for $8 \mathrm{~h}$. Untreated cells were used as blank control. Total RNA was extracted, and cDNA was synthesized using the $\mathrm{RT}^{2}$ First Strand kit (Qiagen $\mathrm{GmbH}$ ) according to the manufacturers' recommendations. The RT ${ }^{2}$ Profiler $^{\mathrm{TM}}$ PCR Array kit 384 (4x96; Qiagen $\mathrm{GmbH}$ ) specific for the human IL-6/STAT3 signaling pathway was used to analyze the mRNA expression levels of 84 associated genes, which included receptors, up and downstream signaling, cytokines and pathway activity in response to the IL-6/STAT3 signaling pathway. The reaction mixture consisted of $650 \mu \mathrm{l} 2 \mathrm{x} \mathrm{RT}^{2} \mathrm{SYBR}$ Green Mastermix (Qiagen $\mathrm{GmbH}), 102 \mu \mathrm{lcDNA}$ and $548 \mu \mathrm{l}$ nuclease-free water to a final volume of $1,300 \mu \mathrm{l}$, and $10 \mu \mathrm{l}$ of this mixture was dispensed in each well of a 96-well plate. RT-qPCR was performed using the 7900HT Fast Real-Time PCR system (Thermo Fisher Scientific, Inc.). The thermocycling conditions consisted of a 
10-min, $95^{\circ} \mathrm{C}$ hot-start, followed by 40 amplification cycles at $95^{\circ} \mathrm{C}$ for $15 \mathrm{sec}$ and $60^{\circ} \mathrm{C}$ for $1 \mathrm{~min}$. The PCR array data was analyzed using the Microsoft Excel 2005 macro program (Microsoft Corporation) and the SABiosciences PCR Array Data Analysis Web Portal (http://SABiosciences.com/pcrarrydataanalysis.php). Each treated group was compared to the blank control. P-values were calculated using Student's t-test on replicate $2^{-\Delta \Delta \mathrm{Cq}}$ values for each gene (38). A total of 5 housekeeping genes, including $\beta$-actin, $\beta 2$-microglobulin, GAPDH, hypoxanthine and ribosomal protein large P0 were used for normalization.

Small interfering (si) RNA transfection. Cells were seeded at a density of 5,000 cells/well in antibiotics-free medium the day before transfection. Lipofectamine ${ }^{\circledR}$ RNAiMAX (Invitrogen; Thermo Fisher Scientific, Inc.) was used as siRNA transfection reagent according to the manufacturer's instructions. Cells grew in medium only and treated with CEBPD-siRNA or Silencer ${ }^{\circledR}$ Select negative control siRNA (5'-UUCUCCGAACGUGUCACGUTT-3') for $24 \mathrm{~h}$ at $37^{\circ} \mathrm{C}$ in a $\mathrm{CO}_{2}$ incubator. A final concentration of $10 \mathrm{nmol}$ siRNA was used after optimization. The siRNA targeting human CEBPD and negative control were designed and synthesized by Shanghai GenePharma Co. Ltd., and the siRNA sequence used in the study was 5'-CCUGGACUUACCACCACUATT-3'.

Establishment of a murine psoriasis model and treatment regimen. A total of 20 male BALB/c mice (20-25 g; 8 -weeks-old) were purchased from the Center of Experimental Animals of China Medical University. All the animals were randomly divided into four groups: i) IMQ group; ii) medium oil (MO) group; iii) shikonin oil (SO) group; and iv) control $(\mathrm{CON})$ group with five mice in each group. All animals were housed in an animal facility with a $12 / 12 \mathrm{~h}$ light/dark cycle at $25 \pm 2^{\circ} \mathrm{C}$ with free access to food and water. All animal experiments were performed in accordance with the National Institutes of Health Guide for the Care and Use of Laboratory Animals and approved by the Animal Care Committee at China Medical University (IACUC no. 16088M)

To induce psoriasis, $50 \mathrm{mg}$ 5\% IMQ (Aldara; iNova Pharmaceuticals Australia Pty Limited) was applied topically on a $2 \times 3 \mathrm{~cm}$ shaved area on the back for 8 consecutive days $(39,40)$. Shikonin (Sigma-Aldrich; Merck KGaA) was dissolved in DMSO and diluted with MO (Fulinmen; COFCO Corporation) at $60^{\circ} \mathrm{C}$ to produce $\mathrm{SO}$ at a final concentration of $0.5766 \mathrm{mg} / \mathrm{ml}$. A $0.5 \mathrm{ml}$ volume SO $(1 \mu \mathrm{M})$ or MO was applied on the affected area $2 \mathrm{~h}$ after each IMQ administration. The severity of the inflamed lesions was evaluated by the Psoriasis Area Severity Index (PASI) (24) that independently scores erythema, scaling and thickening on a scale of 0 to 4 : i) 0 , none; ii) 1 , slight; iii) 2 , moderate; iv) 3 , marked; and v) 4 , very marked. All animals were healthy during the experimental period and sacrificed by cervical dislocation after 8 consecutive days.

Western blot analysis. Mice were sacrificed by cervical dislocation and the lesion areas were shaved. After sterilizing with povidone iodine and $70 \%$ ethanol, skin pieces measuring $2 \times 3 \mathrm{~cm}$ were cut, and the subcutaneous fat and muscle were excised. The tissues were then cut into smaller $0.5 \times 1 \mathrm{~cm}$ sections and digested with $0.25 \%$ trypsin (HyClone; GE Healthcare Life Sciences) for $2 \mathrm{~h}$ at $32^{\circ} \mathrm{C}$. The epidermal layer was detached from the dermis using a sterile scalpel (41). Cells were seeded in 6-well plates, at a density of $5 \times 10^{5}$ cells/well and treated with IL-17, and/or shikonin for $8 \mathrm{~h}$. Untreated cells were used as blank control. Total protein was extracted from cultured cells and epidermal samples using RIPA lysis buffer and quantified with the bicinchoninic acid Protein Assay kit (both Beyotime Institute of Biotechnology). Equal amount of proteins per sample (20 $\mu \mathrm{g}$ and $30 \mu \mathrm{g}$ for cells and tissue, respectively) were separated using 10\% SDS-PAGE, then electro-transferred to PVDF membranes (EMD Millipore). After blocking with $5 \%$ skimmed milk or BSA in TBS + Tween-20 for $1 \mathrm{~h}$ at room temperature, the blots were incubated overnight with rabbit anti-CEBPD (cat. no. ab198230; 1:500), anti-K17 (cat. no. ab109725; $1: 1,000)$ and $\beta$-actin (cat. no. ab8226; 1:8,000) (all Abcam) primary antibodies at $4^{\circ} \mathrm{C}$. The membranes were then incubated with horseradish peroxidase-conjugated goat anti-rabbit IgG (cat. no. ZB-2306; 1:5;000 OriGene Technologies, Inc.) for $1 \mathrm{~h}$ at room temperature. Bands were visualized using an ECL kit (Bio-Rad Laboratories, Inc.) on a MicroChemi $^{\text {TM }}$ Chemiluminescent Imaging System (DNR Bio-Imaging Systems, Ltd.). Band densities were analyzed by ImageJ software (version 1.52a; National Institutes of Health).

Histopathology and immunohistochemistry (IHC). Skin samples from the mice were excised, washed with PBS and fixed in formalin, embedded in paraffin and sectioned as $5 \mu \mathrm{m}$ slices. Then, staining with hematoxylin and eosin (H\&E) was performed, followed by assessment under a light microscope (Olympus Corporation) at a magnification of x200. For IHC, skin samples from the mice were harvested, fixed in $4 \%$ paraformaldehyde at room temperature for $48 \mathrm{~h}$, dehydrated, embedded in paraffin and sectioned (thickness, $5-\mu \mathrm{m}$ ). Gradient ethanol was used to dewax and hydrate the samples, and antigen retrieval was performed in $0.01 \mathrm{M}$ sodium citrate buffer solution $(\mathrm{pH}, 6.0)$ for $25 \mathrm{~min}$ in a water bath at $95^{\circ} \mathrm{C}$. Sections were incubated with anti-CEBPD antibody (1:100; cat. no. ab198230; Abcam) overnight at $4^{\circ} \mathrm{C}$. An IHC kit (Beijing Zhongshan Jinqiao Biotechnology Co., Ltd.) was employed for detection according to the manufacturer's instructions. The sections were incubated with secondary antibody (provided by the kit) at room temperature for $1 \mathrm{~h}$. The sections were incubated with DAB (Beijing Zhongshan Jinqiao Biotechnology Co., Ltd.) for color development and counterstained with hematoxylin at room temperature for $1 \mathrm{~min}$. Three randomly selected regions in the center of each section were assessed under a light microscope (magnification, x400; Olympus Corporation). The mean optical density was obtained by dividing integral optic density by the corresponding area using Image-Pro Plus software (version 6.0; Media Cybernetics, Inc.).

Statistical analysis. Statistical analysis was performed using SPSS v16.0 software (SPSS, Inc.). Grouped pairs were compared using student's t-test. One-way ANOVA was used to compare multiple groups. Fisher's Least Significant Difference (for $\leq 4$ groups) or Tukey's post hoc test $(>4)$ were used to perform inter-group comparisons. $\mathrm{P}<0.05$ was considered to indicate a statistically significant difference. Data are presented as the mean \pm SEM. All assays were repeated three times. 

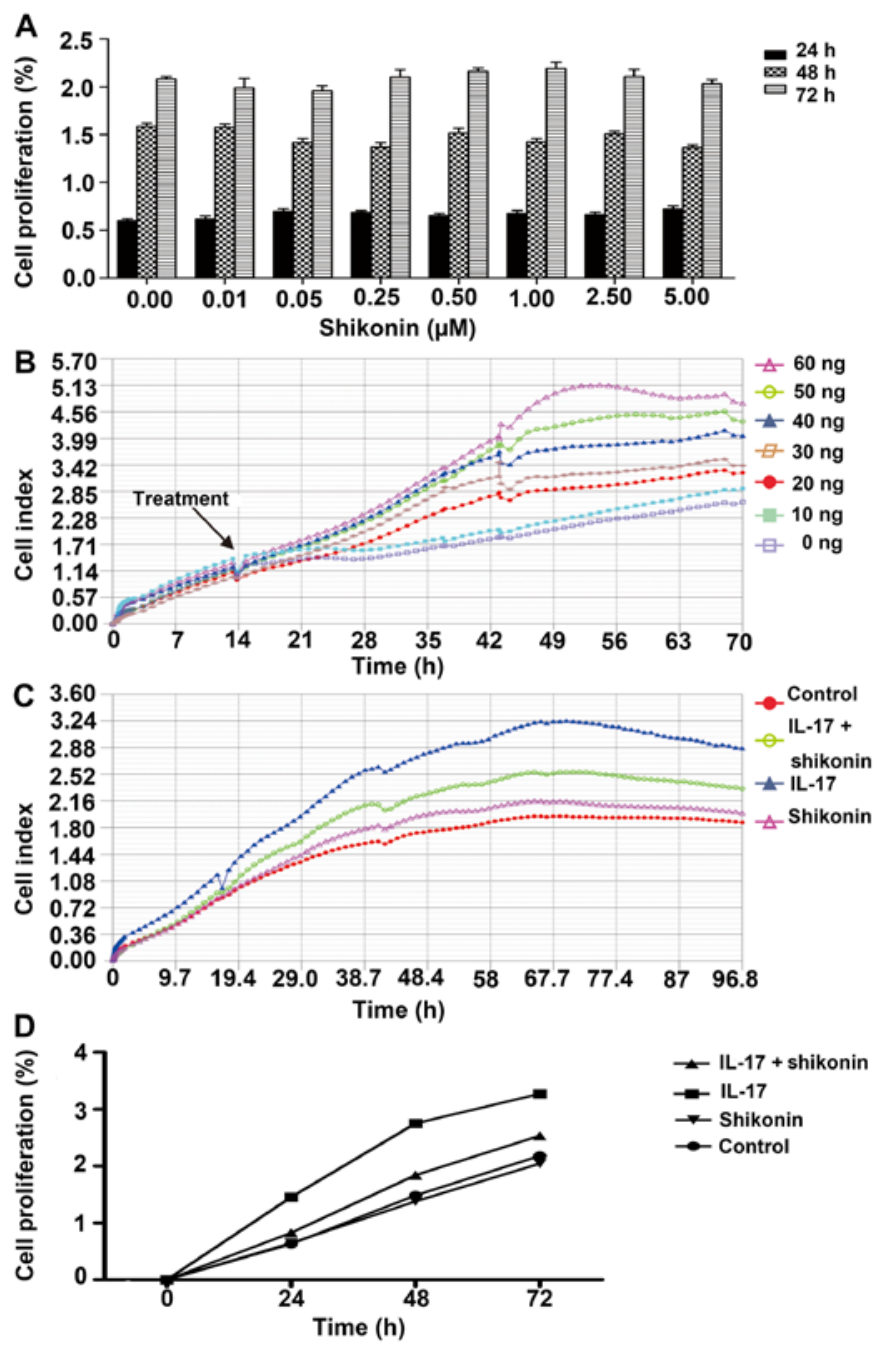

Figure 1. Inhibitory effect of shikonin for IL-17 induced cellular proliferation in HaCaT cells. (A) Optimal concentration of shikonin for the PCR array. The frequency of proliferating $\mathrm{HaCaT}$ cells was evaluated following treatment with $0.01,0.05,0.25,0.5,1,2.5$ or $5 \mu \mathrm{M}$ shikonin for 24,48 and $72 \mathrm{~h}$. Data are presented as the mean \pm SD of six replicates from three independent experiments. (B) Proliferation rates of HaCaT cells treated with different concentrations of IL-17. (C) Proliferation rates of HaCaT cells treated with IL-17 (40 ng/ml), shikonin $(1 \mu \mathrm{M})$ or IL-17 $(40 \mathrm{ng} / \mathrm{ml})+\operatorname{shikonin}(1 \mu \mathrm{M})$. Untreated cells were used as the control. (D) Proliferation rates of HaCaT cells treated with IL-17 and/or shikonin for 24, 48 or $72 \mathrm{~h}$. Data are presented as the mean \pm SEM of six replicates from three independent experiments. IL, interleukin.

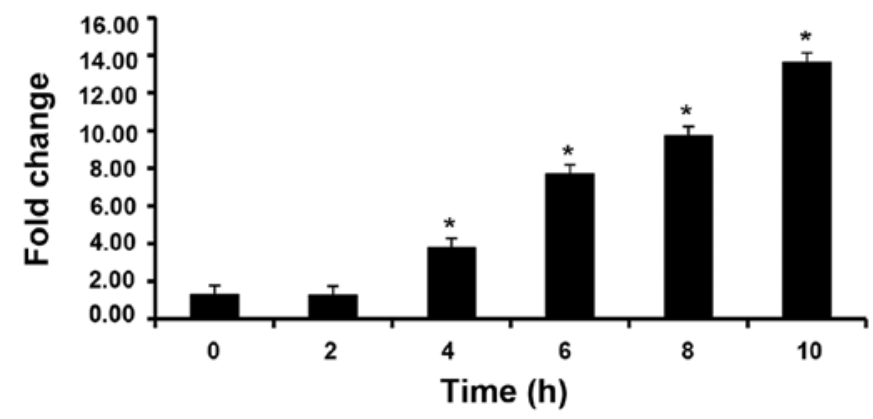

Figure 2. STAT3 mRNA levels in HaCaT cells. HaCaT cells were stimulated with IL-17 (40 $\mathrm{ng} / \mathrm{ml})$ for 2, 4 6, 8 or $10 \mathrm{~h}$. Data are presented as the mean \pm SEM of three experiments. ${ }^{*} \mathrm{P}<0.05$, vs. control.

\section{Results}

Shikonin inhibits IL-17-induced proliferation of keratinocytes. MTS and iCELLigence assays were used to determine the optimal concentrations of shikonin and IL-17. Shikonin had no significant cytotoxic effect on $\mathrm{HaCaT}$ cell proliferation across concentrations ranging from 0 to $5 \mu \mathrm{M}$. Therefore $1 \mu \mathrm{M}$ was used in subsequent experiments (Fig. 1A). The effect of IL-17 on cell proliferation was assessed in real time using the iCELLigence system. IL-17 markedly increased the CI of HaCaT cells in a concentration-dependent manner, compared with the untreated control (Fig. 1B). However, preliminary experiments demonstrated that $1 \mu \mathrm{M}$ shikonin had a good inhibitory effect on IL-17 induced cell proliferation at $\leq 40 \mathrm{ng} / \mathrm{ml}$ (data not shown). Thus, a concentration of $40 \mathrm{ng} / \mathrm{ml}$ was used in subsequent experiments. Shikonin markedly reversed the proliferative effects of IL-17, nearly to same levels as the control group (Fig. 1C). The MTS assay also confirmed the inhibitory effects of shikonin on IL-17-stimulated $\mathrm{HaCaT}$ cells (Fig. 1D). Altogether, these results suggested that IL-17 could promote keratinocyte proliferation and that this effect could be inhibited by shikonin.

Shikonin antagonizes IL-17 by upregulating CEBPD. HaCaT cells stimulated with IL-17 caused a time-dependent significant 
Table I. RT ${ }^{2}$ Profiler PCR array genes expression analysis.

Fold change in expression

\begin{tabular}{llccc} 
& \multicolumn{1}{c}{ Gene name } & IL-17 & Shikonin & IL-17 + Shikonin \\
\cline { 3 - 5 } CCL2 & C-C motif chemokine ligand 2 & -3.34 & $/$ & $/$ \\
CCL4 & C-C motif chemokine ligand 4 & 8.64 & $/$ & $/$ \\
CEBPD & CCAAT/enhancer binding protein $\delta$ & -31.95 & 9.62 & 2.2 \\
CSF1 & Colony stimulating factor 1 & 6.4 & 7.7 & 6.33 \\
CSF2 & Colony stimulating factor 2 & $/$ & 3.4 & $/$ \\
CSF3R & Colony stimulating factor 3 receptor & -5.26 & -14.68 & -23.53 \\
IL11 & Interleukin 11 & $/$ & $/$ & -3.05 \\
IL18R1 & Interleukin 18 receptor 1 & -17.48 & $/$ & -3.34 \\
IL23A & Interleukin 23 subunit $\alpha$ & -5.26 & $/$ & $/$ \\
IL4 & Interleukin 4 & $/$ & 3.25 & 4.07 \\
IL5 & Interleukin 5 & $/$ & 16.51 & $/$ \\
IL6 & Interleukin 6 & $/$ & -11.05 & -3.16 \\
IL6R & Interleukin 6 receptor & $/$ & 5.91 & 5.29 \\
JAK2 & Janus kinase 2 & $/$ & $/$ & -11.07 \\
TLR4 & Toll-like receptor 4 & $/$ & 3.47 & $/$ \\
TNFRSF1B & Tumor necrosis factor receptor superfamily member 1B & $/$ & 9.42 & 8.02 \\
TNFSF10 & Tumor necrosis factor superfamily member 10 & $/$ & $/$ & -3.02 \\
\hline
\end{tabular}

Fold changes in expression between -3 and 3 are indicated by the / symbol.

increase after $4 \mathrm{~h}$ in STAT3 expression. In subsequent experiments, $8 \mathrm{~h}$ was randomly selected as the duration of incubation. (Fig. 2). Therefore, the changes in the expression levels of genes associated with the IL-6/STAT3 pathway in the differentially treated cells using the $\mathrm{RT}^{2}$ Profiler $^{\mathrm{TM}}$ PCR Array were analyzed. As presented in Table I, there were 17 genes, including CCL2, CCL4, CEBPD, CSF1, CSF2, CSF3R, IL11, IL18R1, IL23A, IL4, IL5, IL6, IL6R, JAK2, TLR4, TNFRSF1B and TNFSF10, whose expression levels changed markedly. CEBPD was downregulated in $\mathrm{HaCaT}$ cells in response to IL-17 and upregulated in the presence of shikonin. The fold change in expression levels of CEBPD in shikonin + IL-17 was 2.2. There was no notable difference compared with the blank control group. Similarly, a significant reduction in CEBPD mRNA and protein levels was observed following IL-17 treatment, which was restored in the presence of shikonin (Fig. 3A and C). Thus, it was hypothesized that CEBPD downregulation might represent a proliferative signature in IL-17-stimulated $\mathrm{HaCaT}$ cells. Consistent with this hypothesis, the MTS assay confirmed that when CEBPD is silenced, shikonin does not markedly reverse the proliferative effects of IL-17 (Fig. 3D).

The psoriasis marker, K17 was upregulated in response to IL-17. However, when IL-17 and shikonin were present, there was no notable difference in the mRNA and protein expression levels of K17 compared with the blank control (Fig. 3B and C). When CEBPD was silenced (Fig. 3E and G), K17 was also upregulated in response to IL-17 (Fig. 3F and G). When IL-17 and shikonin were present, the expression levels of K17 in mRNA and protein were upregulated as well (Fig. 3F and G). Collectively, these results suggested that IL-17 could induce proliferation of keratinocytes by downregulating CEBPD, and that the inhibitory effect of shikonin was mediated through CEBPD.

Shikonin alleviates IMQ-induced psoriasis in vivo. The potential therapeutic effects of shikonin were investigated in an IMQ-induced model of psoriasis. Topical application of IMQ resulted in time-dependent thickening, scaling, erythema and inflammation of the affected skin. However, SO treatment markedly lowered PASI scores (Fig. 4A-D), resulting in smoother skin and fewer scales (Fig. 4F). Shikonin-treated mice did not present any changes in their body weight compared to those in the untreated control group (data not shown). MO had no effect on erythema, skin thickening and cumulative PASI scores compared to the untreated psoriatic mice; however, it slightly reduced scaling due to its moisturizing properties. IMQ led to hyperplasia, elongated rete-like ridges and acanthosis in the epidermis, as well as perivascular infiltration of inflammatory cells in the upper dermis similar to that seen in human psoriatic lesions. SO treatment markedly improved these psoriasis-like lesions (Fig. 4E). Consistent with previous studies, which reported aberrantly high K17 levels in early and advanced psoriasis lesions (12), increased in situ K17 expression was detected in the IMQ-induced psoriatic lesions, which was alleviated by shikonin (Fig. 5C). In addition, CEBPD was undetectable in the epidermal keratinocytes and the dermal layer of the IMQ-treated skin (Fig. 5A and B). However, SO restored CEBPD in the epidermis, especially the basal cell layer, to levels similar in the untreated controls (Fig. 5C). These results indicated that shikonin could alleviate IMQ-induced psoriatic lesions in a mouse model, likely through the upregulation of CEBPD. 


\section{A 은}
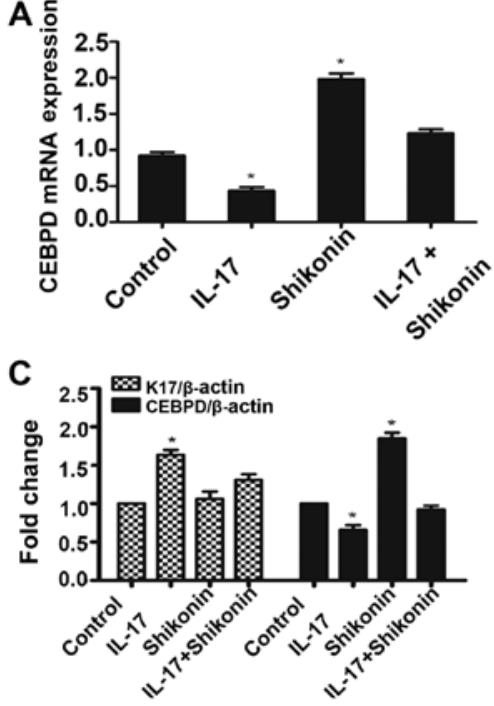

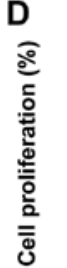
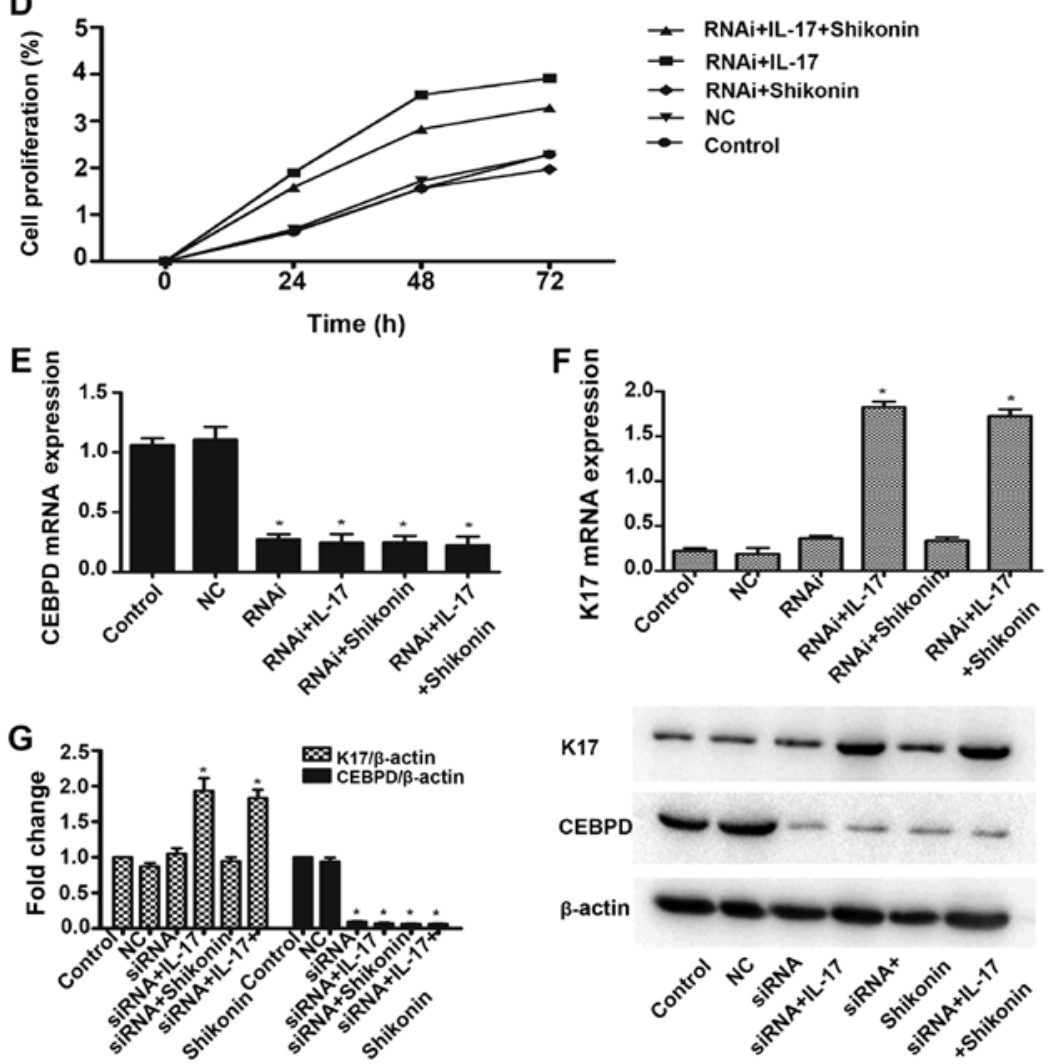

Figure 3. Effects of shikonin on the expression of CEBPD in IL-17-stimulated HaCaT cells. (A) Relative CEBPD and (B) K17 mRNA expression levels in HaCaT cells treated with IL-17 and/or shikonin. (C) Representative western blots for CEBPD and K17 protein levels in HaCaT cells treated with IL-17 and/or shikonin and quantitative analysis. $\beta$-actin was used as the loading control. (D) Proliferation rates of CEBPD-siRNA transfected HaCaT cells treated with IL-17 and/or shikonin for 24, 48 or $72 \mathrm{~h}$. Data are presented as the mean \pm SEM of six replicates from three independent experiments. (E) CEBPD and (F) K17 mRNA expression levels in CEBPD-siRNA cells treated with IL-17 and/or shikonin. (G) Representative western blots for CEBPD and K17 proteins in CEBPD-siRNA cells treated with IL-17 and/or shikonin and quantitative analysis. $\beta$-actin was used as the loading control. ${ }^{*} \mathrm{P}<0.05$, vs. control. IL, interleukin; CEBPD, CCAAT/enhancer-binding protein $\delta$; K17, keratin 17; NC, cells transfected with a negative control scrambled shRNA.

\section{Discussion}

In the present study, shikonin inhibited the proliferative effects of IL-17 on keratinocytes both in vitro and in vivo by targeting the IL-6/STAT3 signaling pathway. Contradictory to previous reports $(33,42)$, in the present study, IL-17 downregulated CEBPD in the hyper-proliferative HaCaT cells, which was reversed by shikonin.
There are six distinct isoforms in the CEBP family, including CEBP $\alpha$, CEBP $\beta$, CEBP $\gamma$, CEBP $\delta$ and CEBP $\varepsilon$ as well as CEBP homologous protein (26). These are involved in the regulation of growth and differentiation of various cells, such as hepatocytes, pneumocytes and hematopoietic cells $(26,43)$. Studies have demonstrated that CEBPD is implicated in cell cycle control: CEBPD mRNA and protein levels are markedly induced in cultured mouse mammary epithelial cells during $\mathrm{G}_{0}$ growth 
A

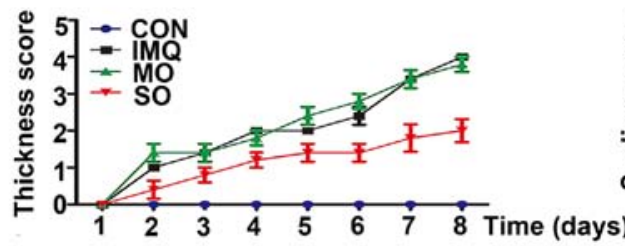

C

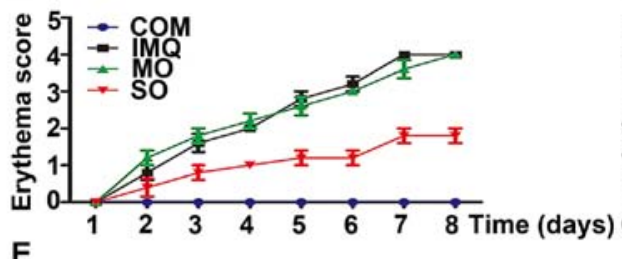

E
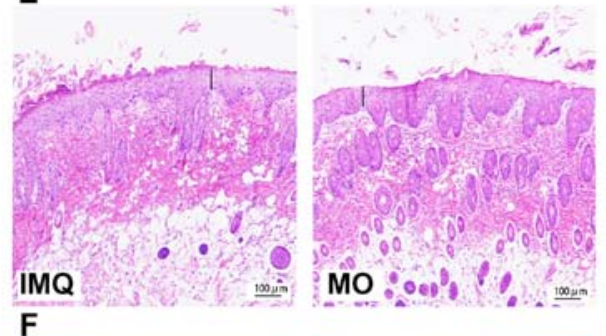

$F$
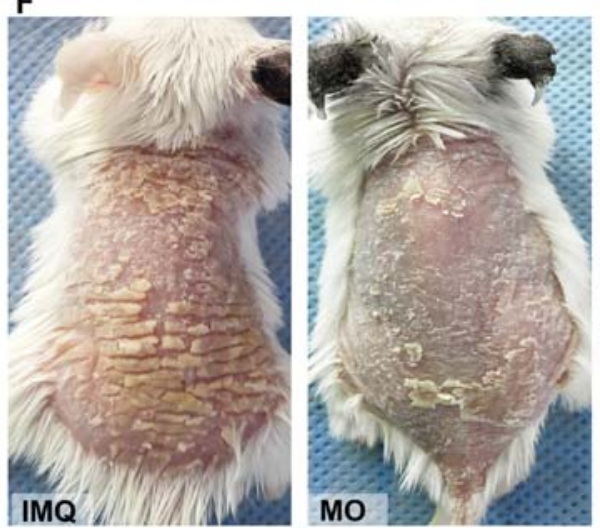

B

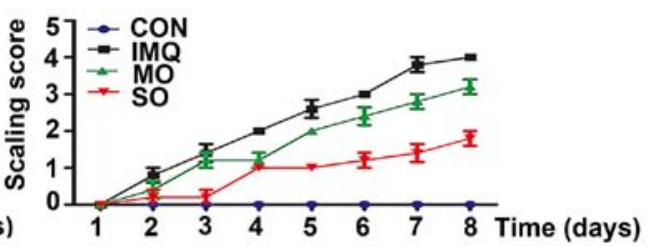

D

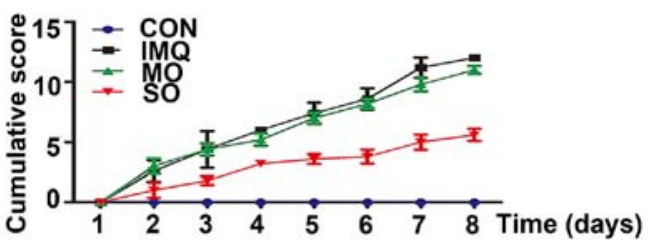

Figure 4. Effects of shikonin in an IMQ-induced psoriatic mouse model. Individual Psoriasis Area Severity Index score for (A) skin thickness, (B) scaling and (C) erythema. (D) Cumulative score in the different experimental groups. Data are presented as the mean \pm SEM. $n=5$. (E) Histological analysis of the affected dorsal skin using hematoxylin and eosin staining. Magnification, x200. (F) Representative images illustrating gross phenotypic changes in the skin lesions after 8 days of treatment. IMQ, imiquimod, SO, shikonin oil; MO, medium oil; CON, control.

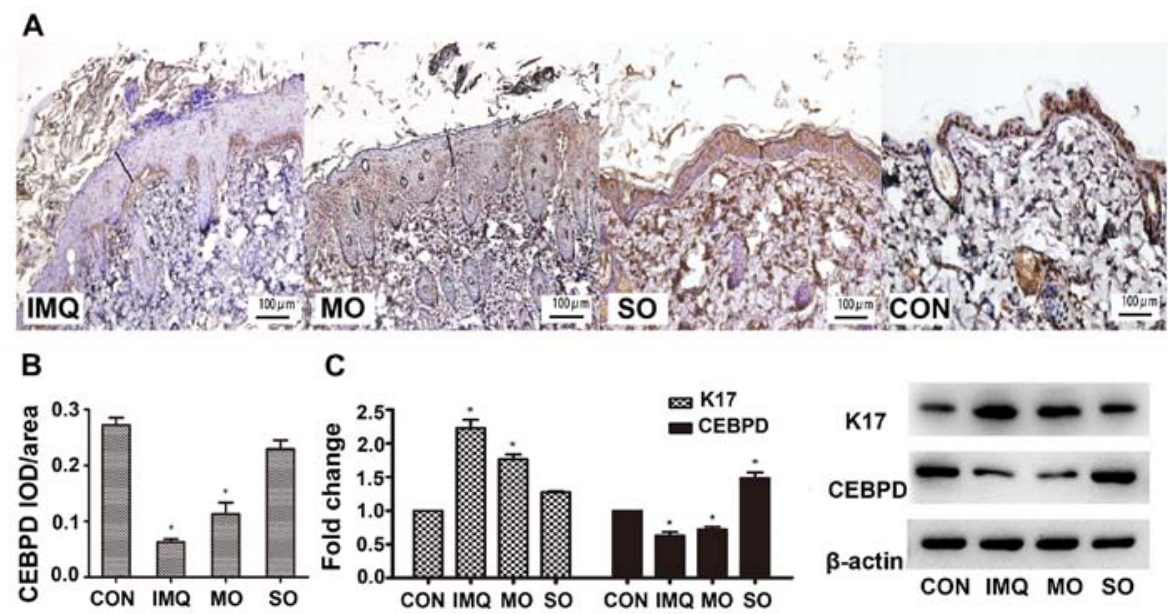

Figure 5. Effects of shikonin on CEBPD expression in an IMQ-induced psoriatic mouse model. (A) Representative IHC images showing in situ expression of CEBPD in the mouse skin. Magnification, x400. (B) Mean optical density of CEBPD. (C) CEBPD and K17 protein expression levels in psoriatic lesions. ${ }^{*} \mathrm{P}<0.05$, vs. CON. CEBPD, CCAAT/enhancer-binding protein $\delta$; K17, keratin 17; IMQ, imiquimod, SO, shikonin oil; MO, medium oil; CON, control; IOD, integrated optical density. 
arrest (44). It also plays an important role in promoting prostate epithelial cell growth arrest and/or apoptosis after androgen withdrawal (43). It has been reported that CEBPD may be induced by many extracellular stimuli, such as IL-1, lipopolysaccharide, interferon (IFN)- $\alpha$, IFN- $\gamma$, and IL-6 $(34,43)$. In addition, Wang et al (33) reported that although CEBPD has long been considered a tumor suppressor gene, CEBPD serves dual roles in pro- and antitumor processes under conditions such as hypoxia and inflammation (34). Furthermore, in human prostate adenocarcinoma LNCaP 104-S and 104-R1 cells, Chuang et al (34) demonstrated that DNA- and histone-mediated epigenetic regulation of CEBPD transcriptional attenuation can occur in a cell type- or tissue-dependent manner. In lesions of patients with psoriasis, keratinocytes are characterized by hyperproliferation and aberrant terminal differentiation and result in the formation of plaque. It is essential for keratinocytes to intrinsic alterations in the response to $\mathrm{T}$ cell-derived signals in psoriasis $(45,46)$. It was hypothesized that the latter would also affect the expression of CEBPD. Thus, lower CEBPD expression induced by IL-17 could lead to excessive proliferation of the HaCaT cells.

CEBPD is a downstream target of p38 (43). A number of studies have suggested that CEBPD transcriptional activation responds to the activation of either STAT3 or p38/CREB (cAMP responsive element binding protein) $(33,47)$. Shan et al $(48)$ demonstrated that shikonin could inhibit cell proliferation and induce apoptosis by modulating phosphorylated (p)-p38/mitogen-activated protein kinase (MAPK), p-JNK and c-Myc. In addition, ERK, JNK and p38 play important roles in shikonin-induced apoptosis $(21,48,49)$. The JAK/STAT3 signaling pathway is involved in psoriasis progression and is also targeted by shikonin to reduce tumor growth and metastasis $(22,50)$. Our previous study demonstrated that shikonin suppressed IL-17-induced, psoriasis-associated cytokines by inhibiting the JAK/STAT3 signaling pathway (23). Several studies suggest that shikonin and its derivatives are effective inhibitors of STAT3, which could be the possible mechanistic basis of the upregulation of CEBPD (21-25). IMQ can induce psoriatic-like plaques in mice by triggering the IL-23/IL-17 axis (46). In the present study, consistent with the in vitro findings, CEBPD was downregulated in IMQ-treated mice and restored by shikonin. Shikonin also alleviated keratinocyte hyperproliferation, inflammatory infiltration and other tissue damage. Thus, shikonin inhibited keratinocyte proliferation and prevented the development of IMQ-induced psoriatic lesions.

K17 is a widely used marker of psoriasis pathogenesis. It is rarely expressed in normal cells but is highly expressed in psoriatic lesions and is upregulated by IFN- $\gamma$, IL-22 and IL-17 (11-13). Yang et al (13) confirmed that under a psoriatic microenvironment with proinflammatory cytokines such as IFN- $\gamma$, the mRNA and protein expression levels of K17 further potentiate the interaction between K17 and STAT3, which subsequently promotes STAT3 phosphorylation, nuclear transport and downstream gene expression levels of cyclin D1. In addition, $\mathrm{K} 17$ promotes the proliferation of psoriatic $\mathrm{T}$ cells and production of cytokines such as IFN- $\gamma$ (51). Our previous study suggested that shikonin downregulated K17 by interfering with STAT3 signaling (24). Similarly, in the present study, shikonin decreased K17 levels in both IL-17-stimulated $\mathrm{HaCaT}$ cells and IMQ-induced psoriasis lesions. In the absence of CEBPD, however, the inhibitory effect of shikonin was impaired, these findings may constitute a potential therapeutic target for psoriasis.

Psoriasis is a complex disease involving keratinocytes, endothelial cells and immune cells, such as macrophages and T lymphocytes $(1,6,10,13)$. Therefore, the effect of shikonin on immune cell infiltration and psoriasis-related cytokine expression requires further investigation. Furthermore, the mechanism of CEBPD regulation in the pathogenesis of psoriasis and the therapeutic effects of shikonin remain to be determined. In summary, shikonin can protect against psoriatic progression and CEBPD might represent a promising therapeutic target in this context.

\section{Acknowledgements}

Not applicable.

\section{Funding}

The present study was supported by The National Natural Science Foundation of China (grant nos. 81673055 and 81402595).

\section{Availability of data and materials}

The datasets used and/or analyzed during the current study are available from the corresponding author upon reasonable request.

\section{Authors' contributions}

XOL, YYX and YJY performed the experiments. HXW, YY and $\mathrm{HG}$ analyzed and interpreted the data. XOL drafted the manuscript. RQQ was involved in statistical analysis and data interpretation. XHG and LG were involved in study conceptualization and obtained funding. All authors have read and approved the final manuscript.

\section{Ethics approval and consent to participate}

The present study was approved by the Animal Care Committee at China Medical University.

\section{Patient consent for publication}

Not applicable.

\section{Competing interests}

The authors declare that they have no competing interests.

\section{References}

1. Di Cesare A, Di Meglio P and Nestle FO: The IL-23/Th17 axis in the immunopathogenesis of psoriasis. J Invest Dermatol 129: 1339-1350, 2009

2. Ramirez-Carrozzi V, Sambandam A, Luis E, Lin Z, Jeet S, Lesch J, Hackney J, Kim J, Zhou M, Lai J, et al: IL-17C regulates the innate immune function of epithelial cells in an autocrine manner. Nat Immunol 12: 1159-1166, 2011.

3. Clark RA: Skin-resident T cells: The ups and downs of on site immunity. J Invest Dermatol 130: 362-370, 2010. 
4. Li Y, Yu C, Zhu WM, Xie Y, Qi X, Li N and Li JS: Triptolide ameliorates IL-10-deficient mice colitis by mechanisms involving suppression of IL-6/STAT3 signaling pathway and down-regulation of IL-17. Mol Immunol 47: 2467-2474, 2010

5. Simanski M, Rademacher F, Schröder L, Schumacher HM, Gläser R and Harder J: IL-17A and IFN- $\gamma$ synergistically induce RNase 7 expression via STAT3 in primary keratinocytes. PLoS One 8: e59531, 2013.

6. Yang L, Li B, Dang E, Jin L, Fan X and Wang G: Impaired function of regulatory $\mathrm{T}$ cells in patients with psoriasis is mediated by phosphorylation of STAT3. J Dermatol Sci 81: 85-92, 2016.

7. Yang XO, Panopoulos AD, Nurieva R, Chang SH, Wang D, Watowich SS and Dong C: STAT3 regulates cytokine-mediated generation of inflammatory helper T cells. J Biol Chem 282: 9358-9363, 2007.

8. Zhou L, Ivanov II, Spolski R, Min R, Shenderov K, Egawa T, Levy DE, Leonard WJ and Littman DR: IL-6 programs $\mathrm{T}(\mathrm{H})-17$ cell differentiation by promoting sequential engagemen of the IL-21 and IL-23 pathways. Nat Immunol 8: 967-974, 2007.

9. Xu L, Kitani A, Stuelten C, McGrady G, Fuss I and Strober W Positive and negative transcriptional regulation of the Foxp3 gene is mediated by access and binding of the Smad3 protein to enhancer I. Immunity 33: 313-325, 2010.

10. Miyoshi K, Takaishi M, Nakajima K, Ikeda M, Kanda T, Tarutani M, Iiyama T, Asao N, DiGiovanni J and Sano S: Stat3 as a therapeutic target for the treatment of psoriasis: A clinical feasibility study with STA-21, a Stat3 inhibitor. J Invest Dermatol 131: 108-117, 2011.

11. Fu M and Wang G: Keratin 17 as a therapeutic target for the treatment of psoriasis. J Dermatol Sci 67: 161-165, 2012

12. Jin L and Wang G: Keratin 17: A critical player in the pathogenesis of psoriasis. Med Res Rev 34: 438-454, 2014.

13. Yang L, Jin L, Ke Y, Fan X, Zhang T, Zhang C, Bian H and Wang G: E3 Ligase Trim21 ubiquitylates and stabilizes Keratin 17 to induce STAT3 activation in psoriasis. J Invest Dermatol 138: 2568-2577, 2018

14. William DJ, Timothy GB and Dirk ME: Andrews' diseases of the skin clinical dermatology. China Science Publishing, Beijing, pp195-198, 2015.

15. Lan W, Wan S, Gu W, Wang H and Zhou S: Mechanisms behind the inhibition of lung adenocarcinoma cell by shikonin. Cell Biochem Biophys 70: 1459-1467, 2014.

16. Gong K, Zhang Z, Chen Y, Shu HB and Li W: Extracellular signal-regulated kinase, receptor interacting protein, and reactive oxygen species regulate shikonin-induced autophagy in human hepatocellular carcinoma. Eur J Pharmacol 738: 142-152, 2014.

17. Gaddipati JP, Mani H, Shefali, Raj K, Mathad VT, Bhaduri AP and Maheshwari RK: Inhibition of growth and regulation of IGFs and VEGF in human prostate cancer cell lines by shikonin analogue 93/637 (SA). Anticancer Res 20: 2547-2552, 2000.

18. Jang SY, Lee JK, Jang EH, Jeong SY and Kim JH: Shikonin blocks migration and invasion of human breast cancer cells through inhibition of matrix metalloproteinase- 9 activation. Oncol Rep 31: 2827-2833, 2014

19. Lu L, Qin A, Huang H, Zhou P, Zhang C, Liu N, Li S, Wen G Zhang C, Dong W, et al: Shikonin extracted from medicinal Chinese herbs exerts anti-inflammatory effect via proteasome inhibition. Eur J Pharmacol 658: 242-247, 2011.

20. Chen X, Yang L, Oppenheim JJ and Howard MZ: Cellular pharmacology studies of shikonin derivatives. Phytother Res 16 : 199-209, 2002

21. Li W, Zhang C, Ren A, Li T, Jin R, Li G, Gu X, Shi R and Zhao Y: Shikonin suppresses skin carcinogenesis via inhibiting cell proliferation. PLoS One 10: e0126459, 2015.

22. Qiu HY, Fu JY, Yang MK, Han HW, Wang PF, Zhang YH, Lin HY, Tang CY, Qi JL, Yang RW, et al: Identification of new shikonin derivatives as STAT3 inhibitors. Biochem Pharmacol 146: 74-86, 2017.

23. Xu Y, Xu X, Gao X, Chen H and Geng L: Shikonin suppresses IL-17-induced VEGF expression via blockage of JAK2/STAT3 pathway. Int Immunopharmacol 19: 327-333, 2014

24. Liu L, Wu Y, Cao K, Xu YY, Gao XH, Chen HD and Geng L: Shikonin inhibits IFN- $\gamma$-induced K17 over-expression of HaCaT cells by interfering with STAT3 signaling. Int J Clin Exp Pathol 8: 9202-9207, 2015

25. Yu YJ, Xu YY, Lan XO, Liu XY, Zhang XL, Gao XH and Geng L: Shikonin induces apoptosis and suppresses growth in keratinocytes via CEBP- $\delta$ upregulation. Int Immunopharmacol 72: 511-521, 2019
26. Ikezoe T, Gery S, Yin D, O'Kelly J, Binderup L, Lemp N, Taguchi $\mathrm{H}$ and Koeffler HP: CCAAT/enhancer-binding protein delta: A molecular target of 1,25-dihydroxyvitamin D3 in androgen-responsive prostate cancer $\mathrm{LNCaP}$ cells. Cancer Res 65: 4762-4768, 2005.

27. Alonzi T, Maritano D, Gorgoni B, Rizzuto G, Libert C and Poli V: Essential role of STAT3 in the control of the acute-phase response as revealed by inducible gene inactivation [correction of activation] in the liver. Mol Cell Biol 21: 1621-1632, 2001.

28. Wang JM, Ko CY, Chen LC, Wang WL and Chang WC: Functional role of NF-IL6 $\beta$ and its sumoylation and acetylation modifications in promoter activation of cyclooxygenase 2 gene. Nucleic Acids Res 34: 217-231, 2006.

29. Ko CY, Chang LH, Lee YC, Sterneck E, Cheng CP, Chen SH, Huang AM, Tseng JT and Wang JM: CCAAT/enhancer binding protein delta (CEBPD) elevating PTX3 expression inhibits macrophage-mediated phagocytosis of dying neuron cells. Neurobiol Aging 33: 422.e11-422.e25, 2012.

30. Tang D, Sivko GS and DeWille JW: Promoter methylation reduces C/EBPdelta (CEBPD) gene expression in the SUM-52PE human breast cancer cell line and in primary breast tumors. Breast Cancer Res Treat 95: 161-170, 2006.

31. Agrawal S, Hofmann WK, Tidow N, Ehrich M, van den Boom D, Koschmieder S, Berdel WE, Serve H and Müller-Tidow C: The $\mathrm{C} / \mathrm{EBP} \delta$ tumor suppressor is silenced by hypermethylation in acute myeloid leukemia. Blood 109: 3895-3905, 2007.

32. Li M, Zhou W, Yuan R, Chen L, Liu T, Huang D, Hao L, Xie Y and Shao J: ROCK2 promotes HCC proliferation by CEBPD inhibition through phospho-GSK3 $3 / \beta$-catenin signaling. FEBS Lett 589: 589, 2015.

33. Wang WJ, Li CF, Chu YY, Wang YH, Hour TC, Yen CJ, Chang WC and Wang JM: Inhibition of the EGFR/STAT3/CEBPD axis reverses cisplatin cross-resistance with paclitaxel in the urothelial carcinoma of the urinary bladder. Clin Cancer Res 23: 503-513, 2017.

34. Chuang $\mathrm{CH}$, Wang WJ, Li CF, Ko CY, Chou YH, Chuu CP, Cheng TL and Wang JM: The combination of the prodrugs perforin-CEBPD and perforin-granzyme B efficiently enhances the activation of caspase signaling and kills prostate cancer. Cell Death Dis 5: e1220, 2014.

35. Sønder SU, Paun A, Ha HL, Johnson PF and Siebenlist U: CIKS/Act1-mediated signaling by IL-17 cytokines in context: Implications for how a CIKS gene variant may predispose to psoriasis. J Immunol 188: 5906-5914, 2012.

36. Yerlikaya A, Erdoğan E, Okur E, Yerlikaya Ş and Savran B: A novel combination treatment for breast cancer cells involving BAPTA-AM and proteasome inhibitor bortezomib. Oncol Lett 12: 323-330, 2016.

37. Türker Sener L, Albeniz G, Dinc B and Albeniz I: iCELLigence real-time cell analysis system for examining the cytotoxicity of drugs to cancer cell lines. Exp Ther Med 14: 1866-1870, 2017.

38. Livak KJ and Schmittgen TD: Analysis of relative gene expression data using real-time quantitative PCR and the 2(-Delta Delta C(T)) Method. Methods 25: 402-408, 2001.

39. Lin YK, Yang SH, Chen CC, Kao HC and Fang JY: Using imiquimod-induced psoriasis-like skin as a model to measure the skin penetration of anti-psoriatic drugs. PLoS One 10: e0137890, 2015 .

40. Van Der Fits L, Mourits S, Voerman JS, Kant M, Boon L, Laman JD, Cornelissen F, Mus AM, Florencia E, Prens EP and Lubberts E: Imiquimod-induced psoriasis-like skin inflammation in mice is mediated via the IL-23/IL-17 axis. J. Immunol 182 . 5836-5845.2009.

41. Morris RJ, Readio N, Boland K, Johnson K, Lad S, Singh A, Singh A, Holtorf S and Skaar S: Isolation of mouse epidermal keratinocytes and their in vitro clonogenic culture. J Vis Exp 10 e58701, 2019.

42. Hu Y, Shen F, Crellin NK and Ouyang W: The IL-17 pathway as a major therapeutic target in autoimmune diseases. Ann N Y Acad Sci 1217: 60-76, 2011.

43. Wang JM, Tseng JT and Chang WC: Induction of human NF-IL6 $\beta$ by epidermal growth factor is mediated through the p38 signaling pathway and cAMP response element-binding protein activation in A431 cells. Mol Biol Cell 16: 3365-3376, 2005.

44. O'Rourke J, Yuan R, DeWille J, O' Rourke JP and Yuan R: CCAAT/enhancer-binding protein-delta $(\mathrm{C} / \mathrm{EBP}-\delta)$ is induced in growth-arrested mouse mammary epithelial cells. J Biol Chem 272: 6291-6296, 1997.

45. Albanesi C, De Pità O and Girolomoni G: Resident skin cells in psoriasis: A special look at the pathogenetic functions of keratinocytes. Clin Dermatol 25: 581-588, 2007. 
46. Chen Y, Yan H, Song Z, Chen F, Wang H, Niu J, Shi X, Zhang D, Zhang N, Zhai Z, et al: Downregulation of TNIP1 expression leads to increased proliferation of human keratinocytes and severer psoriasis-like conditions in an imiquimod-induced mouse model of dermatitis. PLoS One 10: e0127957, 2015.

47. Pan YC, Li CF, Ko CY, Pan MH, Chen PJ, Tseng JT, Wu WC, Chang WC, Huang AM, Sterneck E, et al: CEBPD reverses $\mathrm{RB} / \mathrm{E} 2 \mathrm{~F} 1-\mathrm{mediated}$ gene repression and participates in HMDB-induced apoptosis of cancer cells. Clin Cancer Res 16: 5770-5780, 2010.

48. Shan ZL, Zhong L, Xiao CL, Gan LG, Xu T, Song H, Yang R, Li L and Liu BZ: Shikonin suppresses proliferation and induces apoptosis in human leukemia NB4 cells through modulation of MAPKs and c Myc. Mol Med Rep 16: 3055-3060, 2017.

49. Huang WR, Zhang Y and Tang X: Shikonin inhibits the proliferation of human lens epithelial cells by inducing apoptosis through ROS and caspase-dependent pathway. Molecules 19: 7785-7797, 2014
50. Qiu HY, Zhu X, Luo YL, Lin HY, Tang CY, Qi JL, Pang YJ, Yang RW, Lu GH, Wang XM, et al: Identification of new shikonin derivatives as antitumor agents targeting STAT3 SH2 domain. Sci Rep 7: 2863, 2017.

51. Yang L, Fan X, Cui T, Dang E and Wang G: Nrf2 promotes keratinocyte proliferation in psoriasis through up-regulation of Keratin 6, Keratin 16, and Keratin 17. J Invest Dermatol 137: 2168-2176, 2017.

(i) (9) This work is licensed under a Creative Commons EY No NO Attribution-NonCommercial-NoDerivatives 4.0 International (CC BY-NC-ND 4.0) License. 